\title{
Correction to: Increasing of activity and thermostability of cold active butanol-tolerant endoglucanase from a marine Rhodococcus sp. under high concentrations of butanol condition
}

\author{
Xuhao Zeng ${ }^{1} \cdot$ Dongsheng Xue $^{1}$
}

Published online: 19 July 2018

๑) Springer-Verlag GmbH Germany, part of Springer Nature 2018

Correction to: 3 Biotech (2018) 8:265

https://doi.org/10.1007/s13205-018-1249-4

In the online published article, the acknowledgment section was published incorrectly. The correct acknowledgment is given below:

Acknowledgements This work was supported by Science and Technology Research Project of Hubei Provincial Department of Education (Design of a ethanol-tolerant endoglucanase and ethanol-tolerant mechanism, B2017041).

The original article can be found online at https://doi.org/10.1007/ s13205-018-1249-4.

Dongsheng Xue

xdslfbm@hbut.edu.cn

1 Key Laboratory of Fermentation Engineering (Ministry of Education), Hubei Key Laboratory of Industrial Microbiology, Hubei Provincial Cooperative Innovation Center of Industrial Fermentation, Hubei University of Technology, Wuhan 430068, People's Republic of China 